\title{
CARBON DIOXID IN THE PRIMARY CULTIVATION OF THE GONOCOCCUS
}

\author{
CharLES W. Chapin
}

From the Laboratory of the U. S. Immigration Station, Ellis Island, $N . Y$., and the Laboratory of the U.S. Marine Hospital, Stapleton, N.Y.

In a general investigation of the cultural requirements of the gonococcus it seemed well to test the influence of carbon dioxid at pressures approximating its tension in living tissue, that is, from $5-7 \mathrm{~cm}$. $\mathrm{Hg}$.

For the purpose of this work a gram-negative coccus isolated from the pus of a case of gonorrhea in the male has usually been deemed a gonococcus without further attempt at specific identification. Slight irregularity of contour and more or less variation in size and staining intensity of the individuals, and the transparency or inconspicuousness of the young colonies have been regularly observed characteristics.

For the experiment reported in the table a meat infusion was prepared in the usual way and heated to remove coagulable proteid. To this were added urine, $20 \%$, peptone $0.5 \%$, and a normal dilution of sulphuric acid $1.2 \%$. This mixture was divided in 5 flasks to which were added different percentages of a normal solution of sodium hydroxid as shown in the table, and the reaction readings were taken at this stage of the preparation. The finished medium contained in addition, agar, $q$. s., pea flour $0.5 \%$, dextrose $0.5 \%$, and egg yolk $5 \%$, the yolk being added to the cooled medium just before pouring the plates. Four plates were poured from each flask that the entire series might be duplicated in each of 2 chambers. On the surface of these plates was spread the slightly diluted gonorrheal pus.

The plates were covered with glass covers and placed in two stacks in a pan; each stack was covered by an inverted mouse jar and a little water was poured in the pan to seal the chambers. The carbon dioxid was prepared by treating sodium bicarbonate with sulphuric acid, and collected over water to which a little sodium bicarbonate had.been added. To the atmosphere of one of the jars was added this carbon dioxid, approximately $10 \%$, while the unaltered atmosphere of the other jar served as a control.

After "control" and "carbon dioxid" in the table are shown the readings of the plates on the 2 nd day, the best plates from the standpoint of number of gonococcus colonies being given an arbitrary rating of 10 , obviously inferior plates some lesser numeral, while negative plates are shown by the minus sign.

It appears from a glance at the table that the test plates have been successful at a wide range of reactions while the results in the control series are very inferior. Considering that carbon dioxid forms an acid

Received for publication April 13, 1918. 
on going into solution it might be expected to make an alkaline medium more favorable, acid medium less so, but from the table it appears to have improved the cultural conditions even with highly acid mediums.

This table is a selection from the records of several somewhat similar experiments with mediums containing raw proteid (egg yolk, ascitic fluid, calf serum). The medium and other technical details have been varied in numerous ways, and the pus has been obtained from several different patients during the course of the experiments. The results have been consistent as to the main issue though it has occurred that the control plates have been fairly successful at a limited range of reactions, thus obscuring comparison at the acid end of the series.

TABLE 1

Results of Experiments to Show the Value of $\mathbf{C O}_{2}$ in the Cultivation of the Gonococcus

\begin{tabular}{|c|c|c|c|c|c|}
\hline Normal NaOH, per cent.................... & 0 & 1.2 & 2.3 & 3.7 & 4.7 \\
\hline 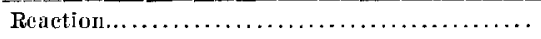 & 3.4 & 2.6 & 1.8 & 0.5 & alk \\
\hline 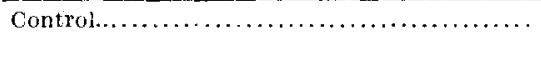 & - & $\begin{array}{l}1 \\
1\end{array}$ & $\begin{array}{l}1 \\
2\end{array}$ & $\overline{-}$ & - \\
\hline 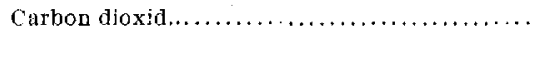 & $\begin{array}{l}3 \\
3\end{array}$ & $\begin{array}{l}10 \\
10\end{array}$ & $\begin{array}{l}10 \\
10\end{array}$ & $\begin{array}{r}10 \\
8\end{array}$ & $\begin{array}{l}10 \\
10\end{array}$ \\
\hline
\end{tabular}

There remains to be considered the theoretical possibility that carbon dioxid might improve even an acid medium by its independent effect on the reaction of the raw proteid, which may not have assimilated with the reaction of the rest of the medium at the temperature. $\mathrm{My}$ experiments on this point have shown distinct evidence that the carbon dioxid improves such meditums by some effect on the raw proteid constituent, though the data on this point are still insufficient.

Wherry and Oliver ${ }^{1}$ have shown that the gonococcus grows best at an oxygen pressure below that of the air, such condition being obtained in most of their experiments by the atmospheric activity of $B$. subtilis. It would seem that the general principle of their technic might easily be adapted to enrich the atmosphere with carbon dioxid at the same time. A lighted candle left in the jar beside the plates at the time of sealing the chamber, I have found a convenient means of establishing a highly favorable atmosphere.

To summarize, an atmosphere rich in carbon dioxid has been found to facilitate the primary cultivation of the gonococcus on mediums containing raw proteid.

1 Jour. Infect. Dis. 1916, 19, p. 288. 